\title{
METHODOLOGY FOR MANAGING CLAIMS AND DISPUTES IN CONSTRUCTION PROJECTS
}

\author{
Esraa Elsaid Ibrahim Elsayad, Mahmoud Ahmed Zaki , Hosny Ahmed Dewer and \\ Architecture and Head of Department of Architecture - Tanta University) \\ Esraa hany94@yahoo.com, hishadly@gmail.com
}

\begin{abstract}
ABSTRAC
Contracts for construction projects are defined as the agreement between the employer (owner) and the contractor (contractor) through specific contractual procedures that define each of the responsibilities of the parties to reach the desired goal of the project. This relationship becomes complex and complex and the projects become more complex, ambiguous and obscured. To the obligations of each of the different project parties, which directly affect the rate of implementation, cost and success of those projects that must be characterized by cooperation and understanding. Many disputes may arise because of poor drafting, complex and inaccurate terminology or failure to address the terms of the contract to determine the obligations and rights of each contracting party, which may cause a dispute between the contracting parties about a person's eligibility for a claim or his obligation to perform a particular job, Where the claim is defined as a claim based on the provisions of the contract, law or custom confirming the entitlement of a person before another person based on a particular fact as a result of a breach of any of the various contract obligations, and the most common claims are by the contractor and an additional time period or additional material cost or right In time and additional cost. These claims arise on different grounds for a variety of reasons and are taken in many stages through different stages of the contract.This success depends on how to manage the disputes and claims that occur on the project during its various stages, by following a scientific methodology considered in light of the contract and the law to reach peaceful solutions to avoid these claims before reaching more complex stages of arbitration and legal issue to achieve the success of the project on time Cost and quality required.
\end{abstract}

Keywords : Claims Process ; Dispute Management Program ; Negotiation ; Arbitration ; Egyptian Code ; FIDIC contract.

$$
\text { منهجيه لإدارة المطالبات والمناز عات فى مشروعات التشييد }
$$

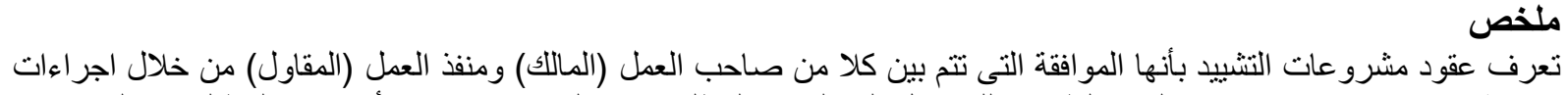

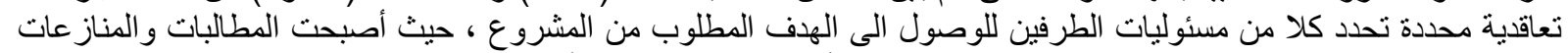

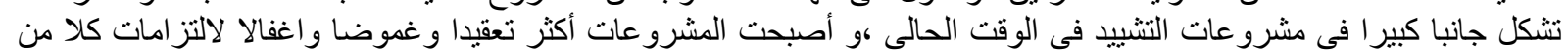

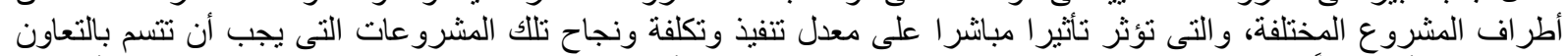

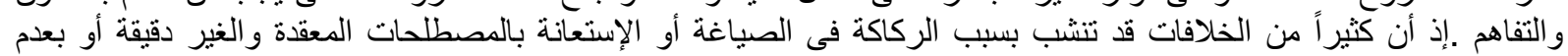

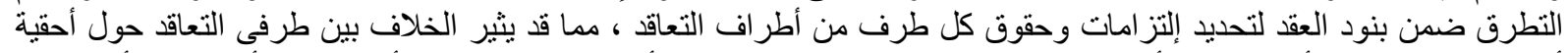

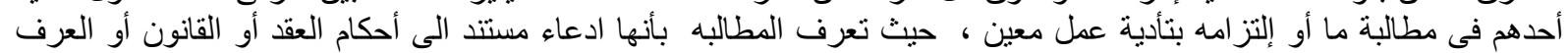

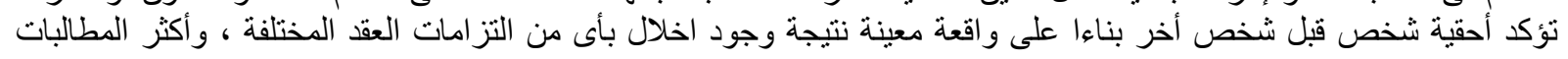




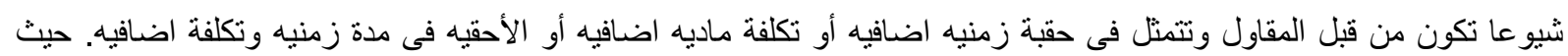

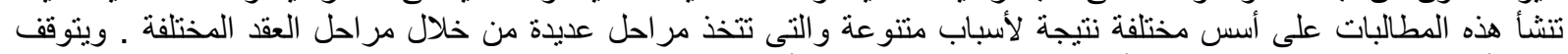

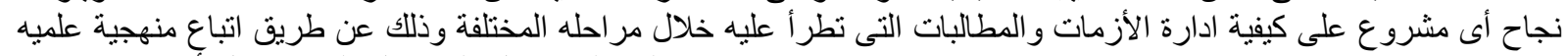

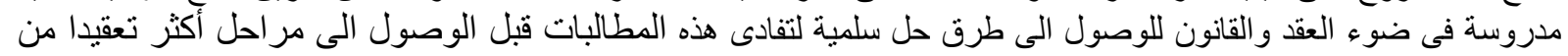

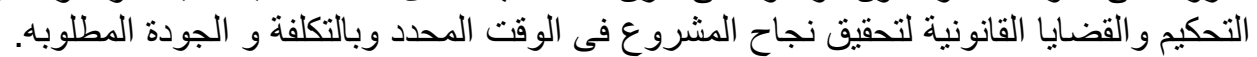

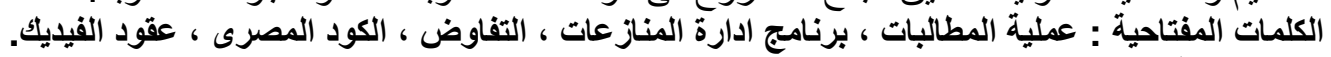

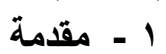

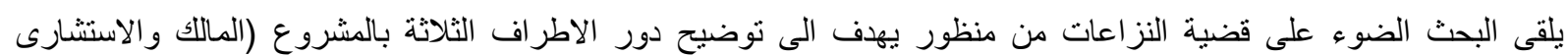

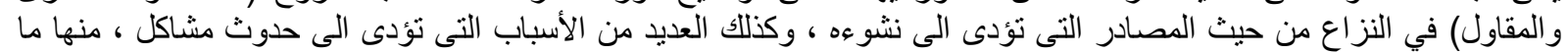

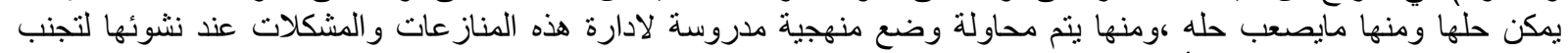

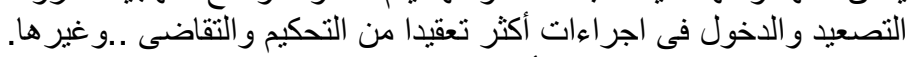

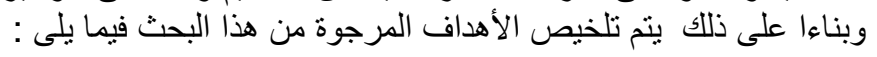

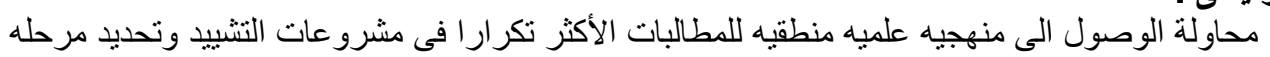

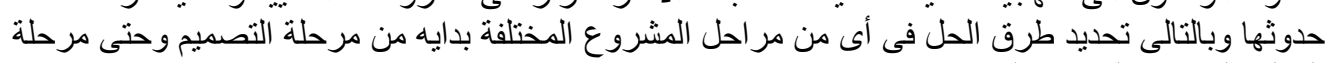

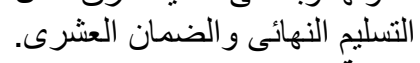

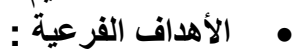
ـ التعرف على الأسباب الأكثر شيو عا للمطالبات.

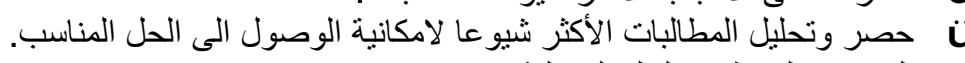

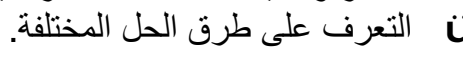

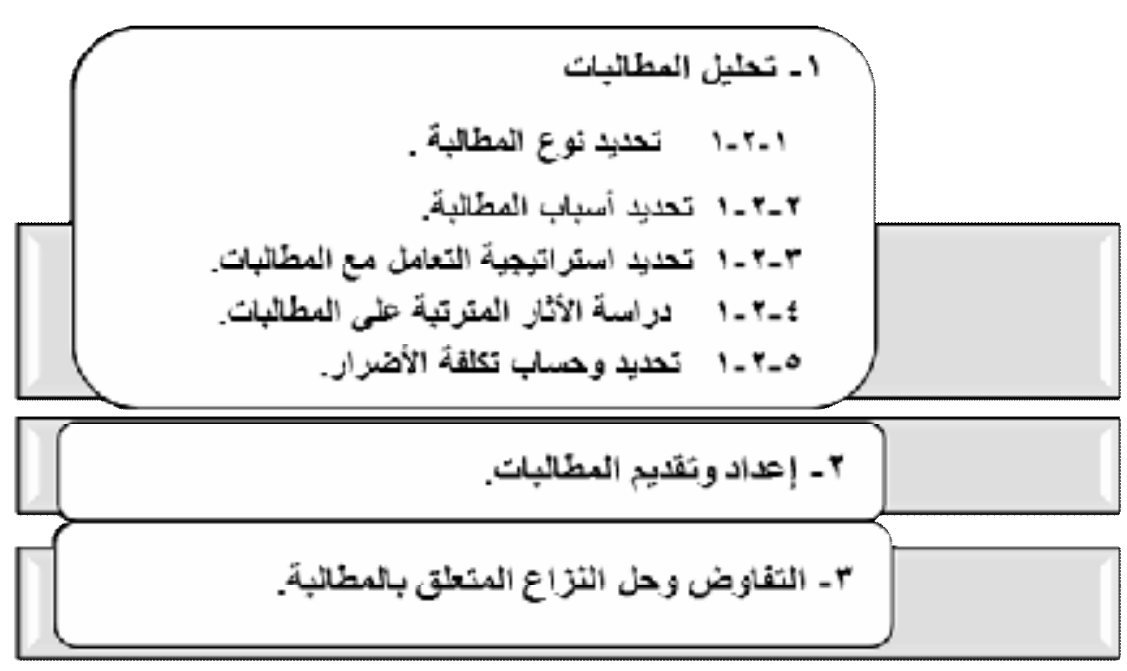

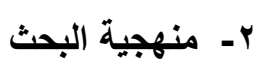

شكل (1) يوضح الألية المتبعة فى تناول المطالبات والمنازعات فى مشروعات التشييد

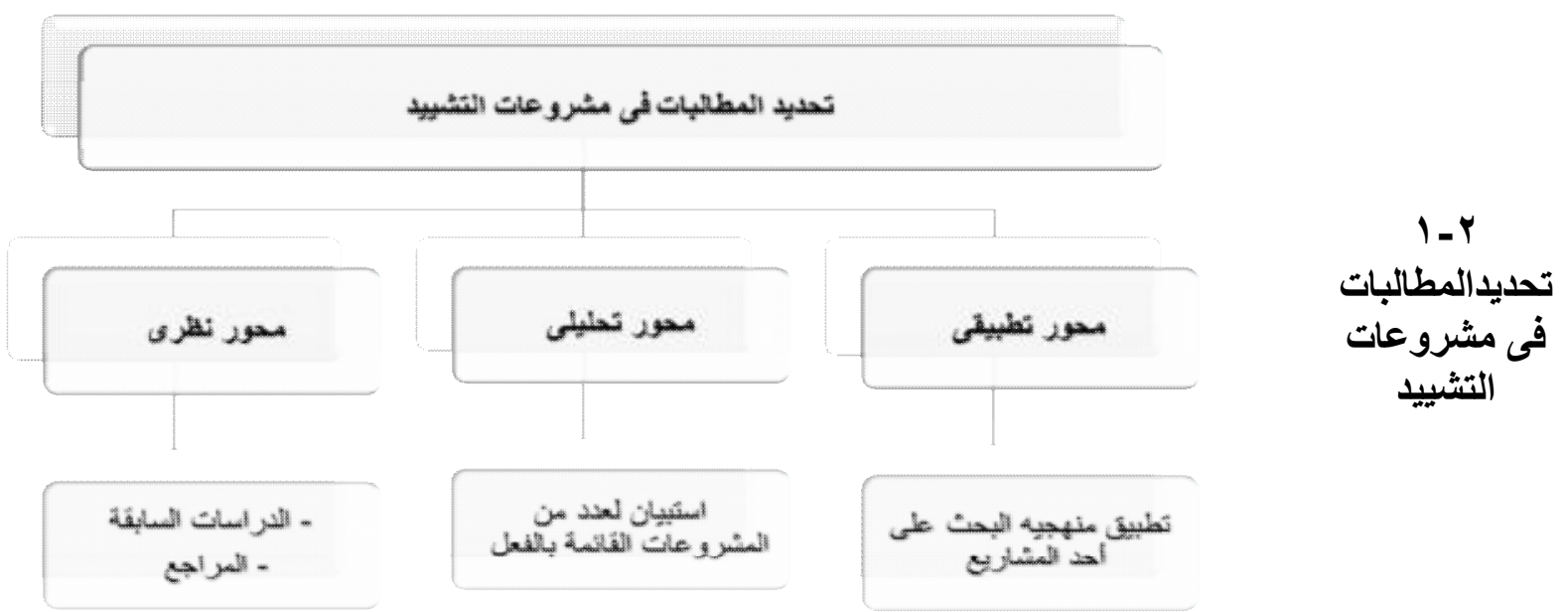

شكل (ץ ) يوضح ألية تحديد المطالبات فى مشروعات التشييا 


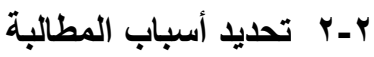

\begin{tabular}{|c|c|}
\hline اسباب المطالبات والنزاعات فى مشروعات التشييد & 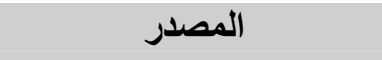 \\
\hline 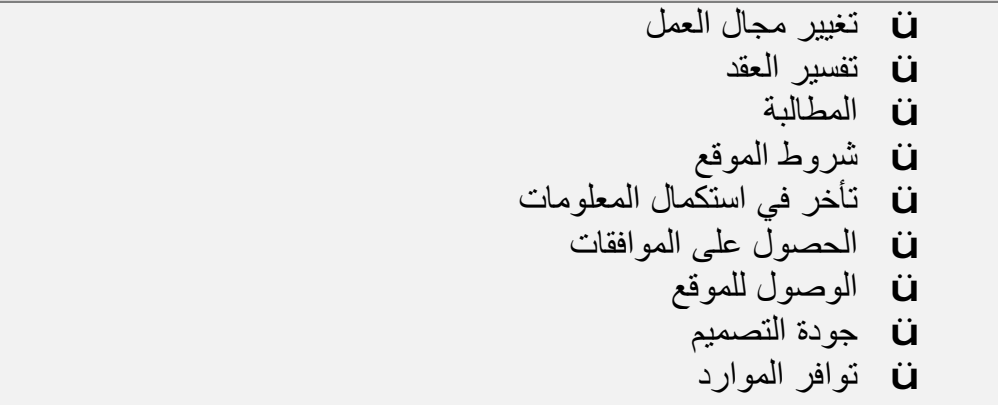 & $\begin{array}{c}\text { Black Dawson waldrou } \\
\text { (2006) }\end{array}$ \\
\hline 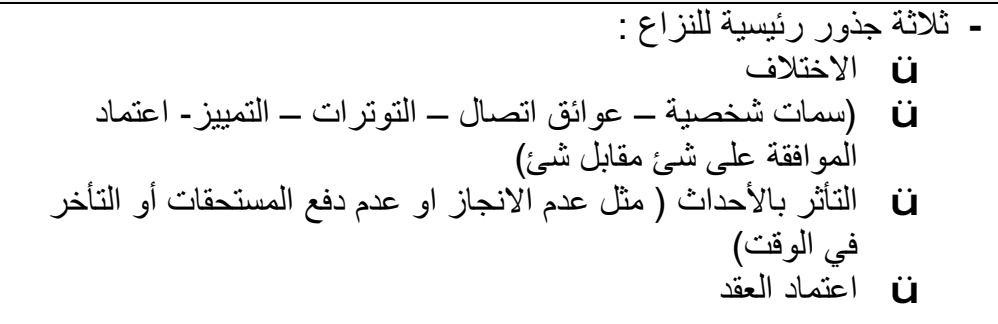 & Cheung and Yui (2006) \\
\hline 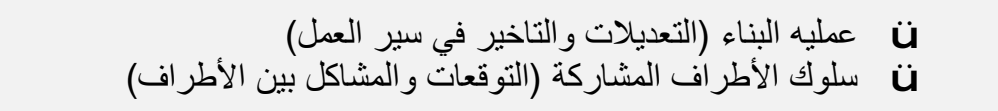 & Yiu and Cheung (2004) \\
\hline 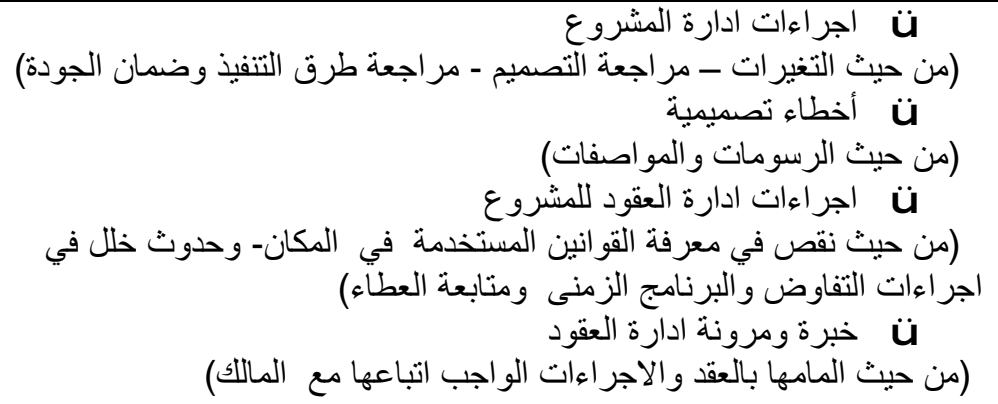 & Killian (2003) \\
\hline
\end{tabular}

جذول ( ) أسباب المطالبات والمناز عات في مشروعات التثييد

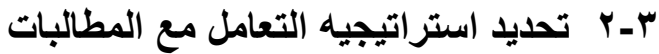

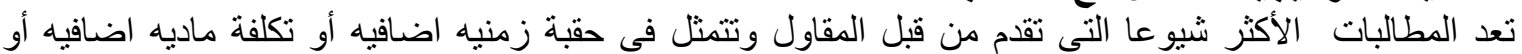

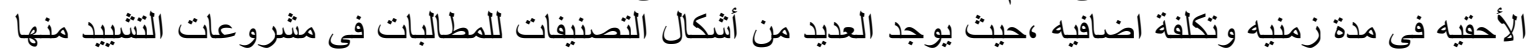

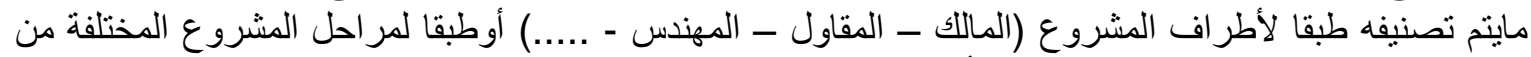

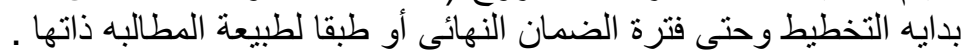

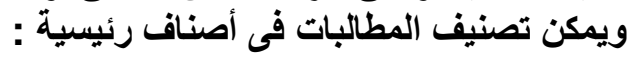

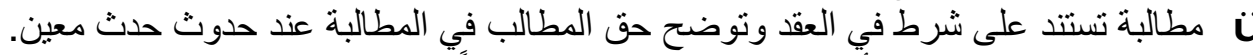

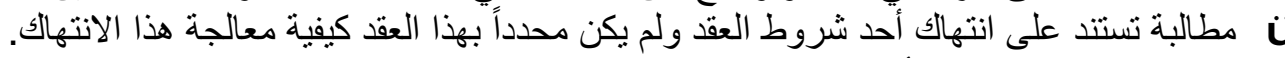
د مطالبة تستند على قانون أو لائحة بمجال التشئيد.

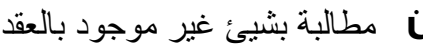




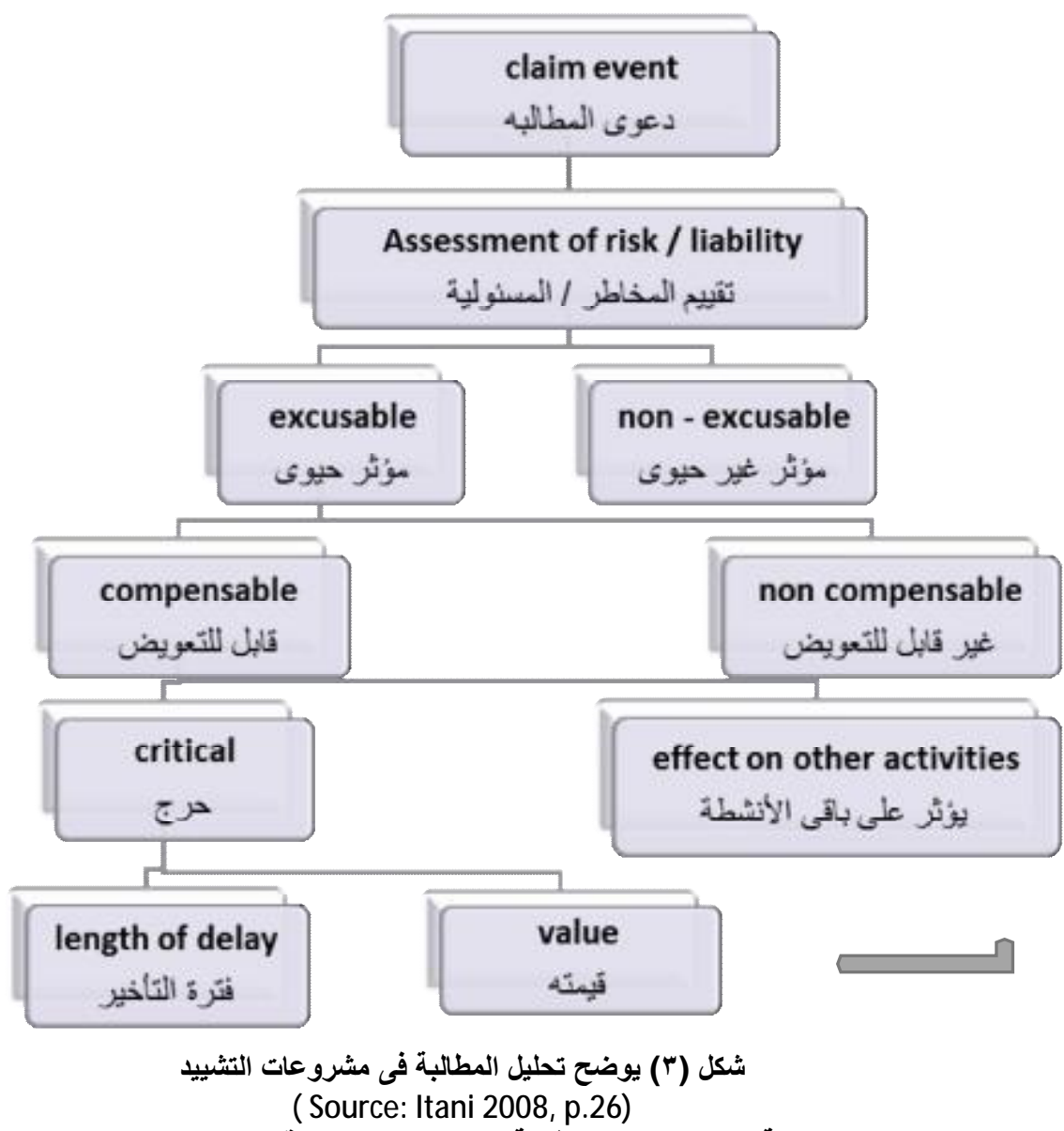

هذا ويعتمد تحديد نوع وحجم المعالجة عندما تكون المطالبة مستندة على سند قوي حسب نوع العقد وما يسمح به، والتى تتحكم فيها العوامل التالية:

الطرف المنفذ للمعالجة. أسلوب تنفيذ المعالجة.

تكلفة المعالجة وبيان الطرف المعنى بالمعالجة.

\begin{tabular}{|c|c|c|c|c|}
\hline $\begin{array}{l}\text { Type of cost claimed } \\
\text { نوع التكلفة المطالب بها }\end{array}$ & $\begin{array}{l}\text { Delay } \\
\text { claim } \\
\text { clailiالبيه }\end{array}$ & $\begin{array}{l}\text { Scope of } \\
\text { work claim } \\
\text { نطالم العمل بالبه }\end{array}$ & $\begin{array}{l}\text { Acceleration } \\
\text { claim } \\
\text { التعجيل بالمطالبه }\end{array}$ & $\begin{array}{l}\text { Changing } \\
\text { site } \\
\text { condition } \\
\text { حاله الموقه بتغيير }\end{array}$ \\
\hline $\begin{array}{l}\text { Additional direct labor hours } \\
\text { ساعات العمل المباشرة الاضافيه }\end{array}$ & & $\mathrm{X}$ & & $\mathbf{X}$ \\
\hline $\begin{array}{l}\text { Additional direct labor hours due to lost } \\
\text { productivity } \\
\text { ساعات العمل الاضافيه بسبب فقدان الانتاجيه }\end{array}$ & $\mathrm{X}$ & $\mathrm{O}$ & $X$ & O \\
\hline $\begin{array}{l}\text { Increase labor rate } \\
\text { زيادة معدل العمالة }\end{array}$ & $X$ & $\mathrm{O}$ & $X$ & O \\
\hline $\begin{array}{l}\text { Additional material quality } \\
\text { كميه المواد الاضافيه }\end{array}$ & & X & $\mathrm{O}$ & $\mathbf{O}$ \\
\hline $\begin{array}{l}\text { Additional material unit price } \\
\text { سعر المو اد الاضافيه }\end{array}$ & $X$ & $X$ & $\mathrm{O}$ & O \\
\hline $\begin{array}{l}\text { Additional subcontractor work } \\
\text { الأعمال الاضافيه من قبل مقاولى الباطن }\end{array}$ & & $X$ & & O \\
\hline
\end{tabular}




\begin{tabular}{|c|c|c|c|c|}
\hline $\begin{array}{l}\text { Additional subcontractor cost } \\
\text { التكلفة الاضافيه من قبل مقاولى الباطن }\end{array}$ & X & $\mathrm{O}$ & $\mathrm{O}$ & $\mathbf{X}$ \\
\hline $\begin{array}{l}\text { Equipment rental cost } \\
\text { تكلفة تأجير المعدات }\end{array}$ & $\mathrm{O}$ & $X$ & $\mathrm{X}$ & $\mathbf{X}$ \\
\hline $\begin{array}{l}\text { Cost for owned equipment use } \\
\text { تكلفة استخدام المعدات المملوكة }\end{array}$ & X & $\mathrm{X}$ & $\mathrm{O}$ & $\mathbf{X}$ \\
\hline $\begin{array}{c}\text { Job overhead costs (variable) } \\
\text { التكاليف المتغيرة لكافة الأعمال }\end{array}$ & $\mathrm{O}$ & $\mathrm{X}$ & $\mathrm{O}$ & $\mathbf{X}$ \\
\hline $\begin{array}{l}\text { Interest or finance costs } \\
\text { تكاليف التمويل / الفو ائد }\end{array}$ & $X$ & $\mathrm{O}$ & $\mathrm{O}$ & O \\
\hline Profit الربح & $\mathrm{O}$ & $X$ & $\mathrm{O}$ & $\mathbf{X}$ \\
\hline $\begin{array}{l}\text { Loss of opportunity profit } \\
\text { تقليل أو خسارة فرص الربح }\end{array}$ & $\mathrm{O}$ & $\mathrm{O}$ & $\mathrm{O}$ & O \\
\hline
\end{tabular}

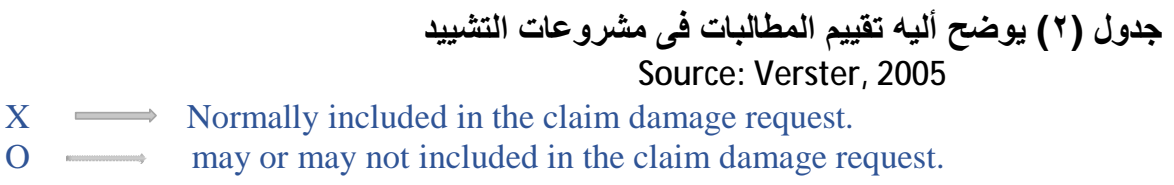

\section{1-}

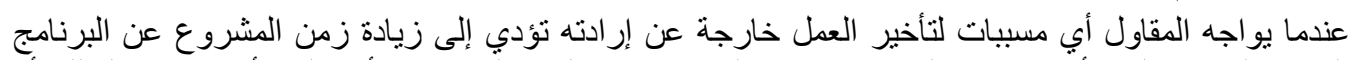

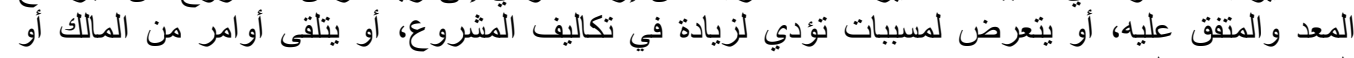

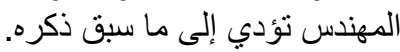

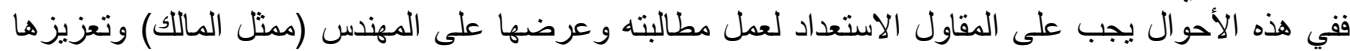

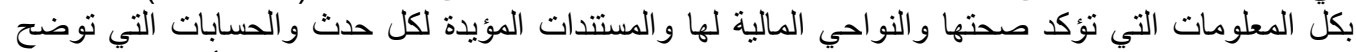
للمهندس ما تكبده المقاول من تكاليف زائدة عن المخطط حتى يقوم المهندس بدر استها وتحديد أحقية المقاول لهات المات

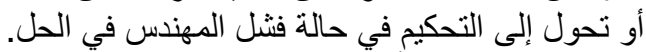

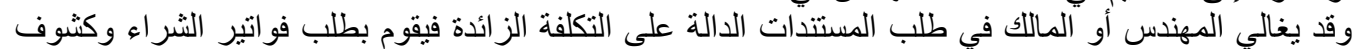

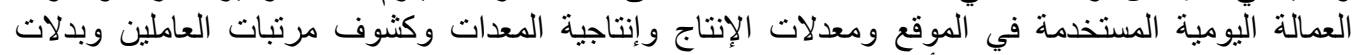

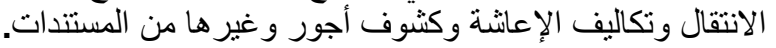

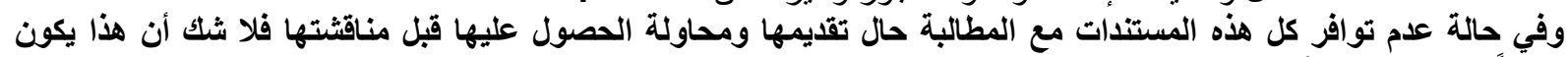

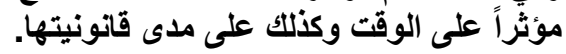

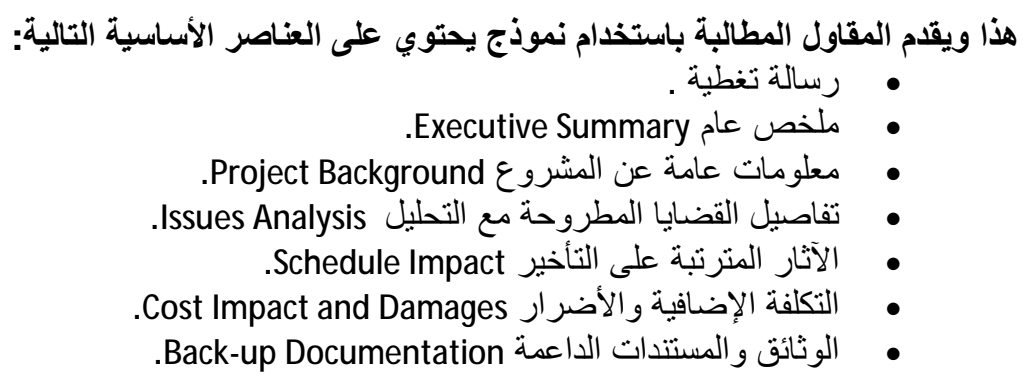

وكمثال على الوثائق والمستندات التي يمكن استخدامها لاى تقديم المطالبة موضح أدناه بعضاً من هذه النماذج.

\section{- The most important records to maintain are:}

- Timecards.

- Personal daily diaries.

- Superintendent's and Inspectors' Daily Reports.

- Correspondence: letters, facsimiles, transmittals, memos,

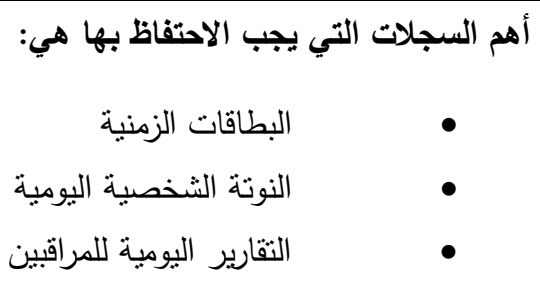

$$
\text { والمسئولين }
$$


telephone conversation notes (telcons), e-mail, etc.

- Submittals (shop drawings, catalog cuts, etc).

$$
\text { التسليمات (مخططات، }
$$

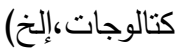

- Requests for Information (RFIs).

- Extra Work Orders (EWOs).

$$
\begin{aligned}
& \text { طلب معلومات إضافية } \\
& \text { أوامر الأعمال الإضافية } \\
& \text { السجلات: النسليمات ، طلب المعلومات، } \\
& \text { أوامر الأعمال الإضافية، الأعمال الإضافية، ... }
\end{aligned}
$$

- Logs: submittals, RFIs, notice of change, change order proposals, change orders, etc.

- Schedules (your own and the GC's, if you are a subcontractor).

- Meeting Minutes and Notes (your own, and ask for corrections for errors in the minutes of meeting).

- Photographs.

الجداول الزمنية للمقاول الرئيسي والفرعي

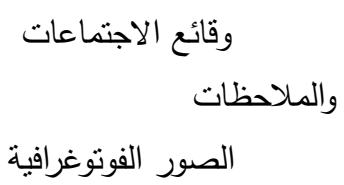

\section{RECORDKEEPING شكل (؛ ) مستندات المطالبة Source: (FIDIC contract ,1999)}

وبعض الشروط العامة للتعاقد مثل (الفيديك وشروط البنك الدولي) تلزم المقاول بمجرد حدوث الحدث المؤثر على وقته أو تكلفته

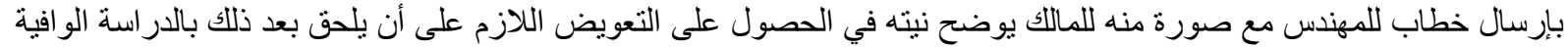
لل المطالبات وكذللك المستتدات الدالة عليها. (أنظر الشكل ه).

\begin{tabular}{|c|c|c|c|c|}
\hline المطالبة رقم (؛) & المطالبة رقم (r) & المطالبة رقم (Y) & المطالبة رقم (1) & \\
\hline رب العمل (الماللك) & المقاول & المقاول & المقاول & الطرف القائم بالمطالبة \\
\hline 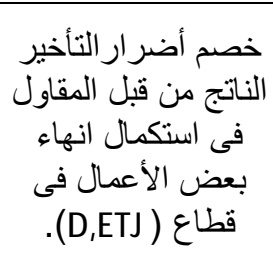 & 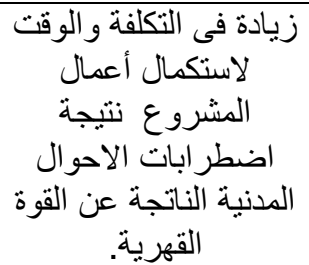 & الوق الوقود في الرمادات & 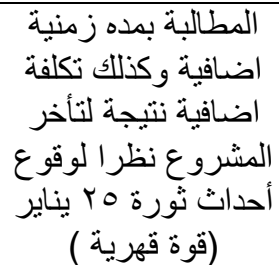 & بيان المطالبة \\
\hline
\end{tabular}

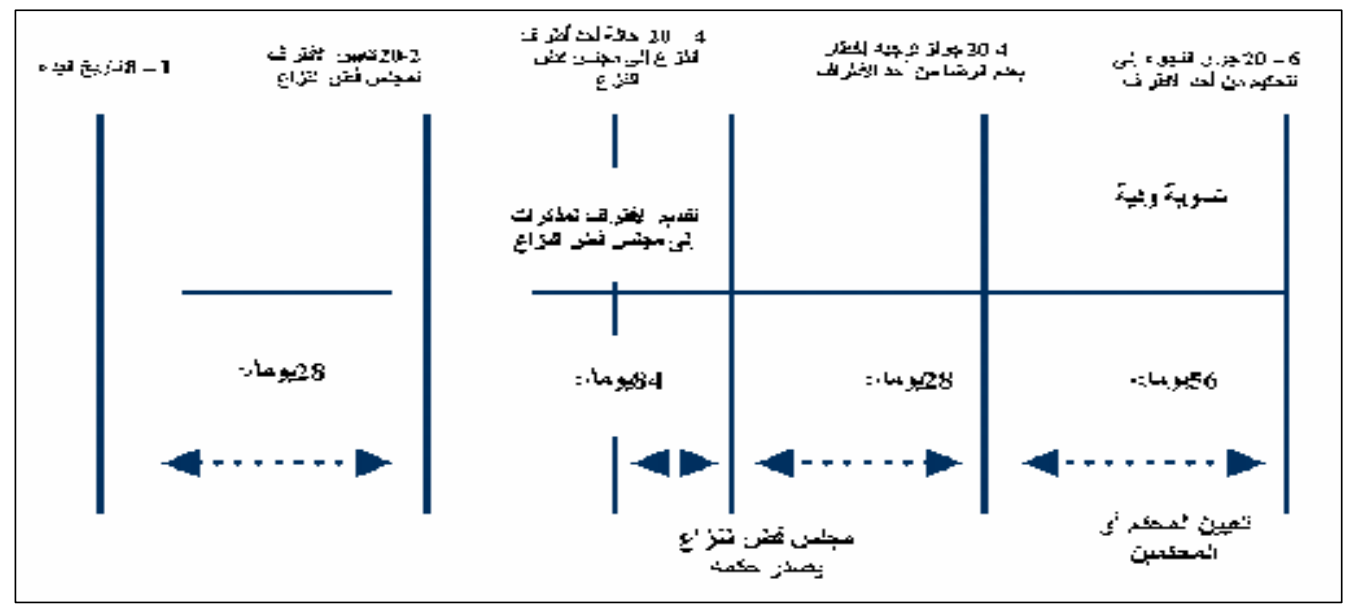

شكل (0) يوضح أليه تقديم المطالبات طبقا لعقود الفيديك

(Source: Itani, 2009, p.25)

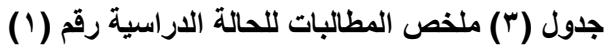


منهجيه لإدارة المطالبات و المناز عات فى مشرو عات التشييد

\begin{tabular}{|c|c|c|c|c|}
\hline 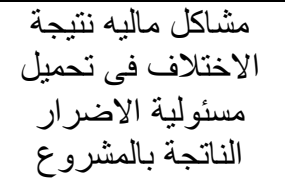 & (تأخير الموارة الققة على المقل & 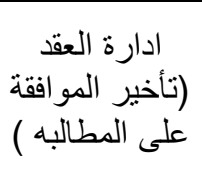 & (تأخير الموافقة على العقد & مصدر المطالبة \\
\hline الأضرار طبل مسئولية لشروط العبرة & تكلفة اضافية & تكلفة اضافية & زكلفة اضنافية ومدة & النتائج \\
\hline - & & ع ال يوم & مب يوم & مطالبة بمده زمنية اضافية \\
\hline ) (^.......... & 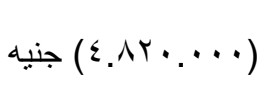 & جنيه & (...... (Y) جنيه & مطالبة بتكلفة اضافية \\
\hline لم يتم تسويتها & لم يتم تسويتها & لم يتم تسويتها & لم يتم تسويتها & كيفية التعامل مع المطالبة \\
\hline & & & & التعويضات الاضافية فى الزمنية \\
\hline & & & & التعويضات الاضافية فى المدة الزمنية \\
\hline
\end{tabular}

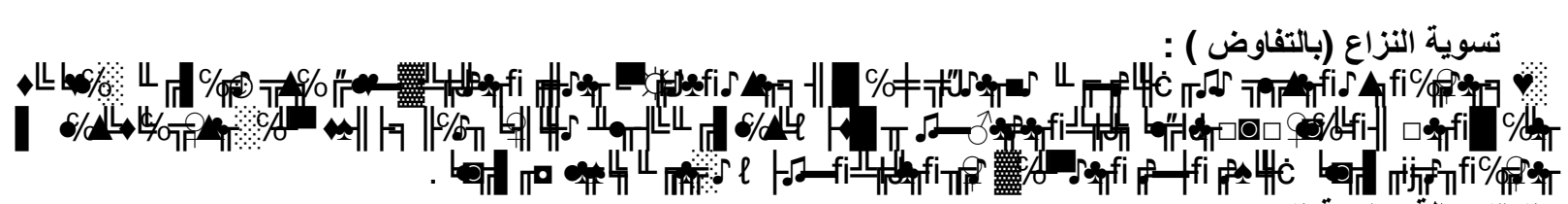

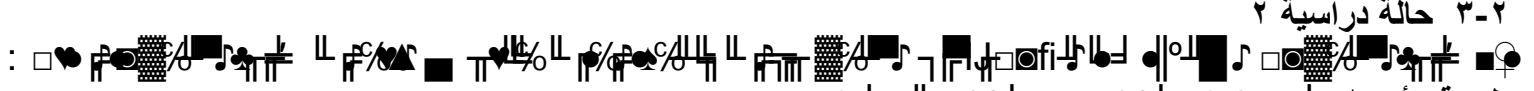
طيعة الأعمال :

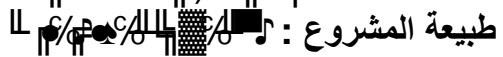

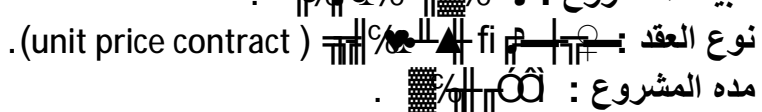

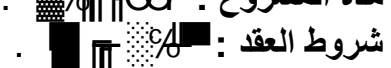
وتتلخص المناز عات الخاصة بالمشروع فيما يلى طبقا للجدول رقم (؛ ):

جدول (؛) ملخص المطالبات للحالة الدراسية رقم (r)

\begin{tabular}{|c|c|c|c|}
\hline المطالبة رقم (r) & المطالبة رقم (Y) & المطالبة رقم (1) & \\
\hline المقاول الرئيسى & مقاول الباطن & مقاول الباطن & الطرف القائم بالمطالبة \\
\hline تطبيق التعويضات الناتجة الأنتاء من الأنهاء & 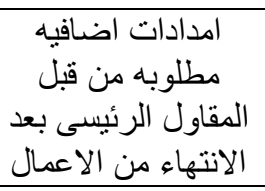 & 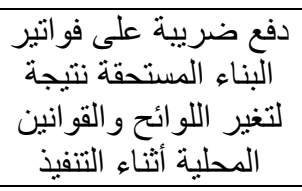 & بيان المطالبة \\
\hline مسئولية الاضتلاف في تحمبل ماليل نتيجة & 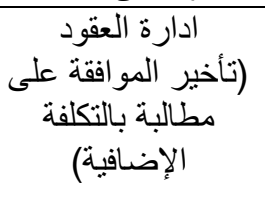 & أثتحداث قو انين جديدة & مصدر المطالبة \\
\hline تكلفة ومدة زمنيه اضافيه & تكلفة اضافية & تكلفة اضافية & النتائج \\
\hline 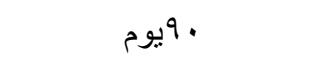 & - & 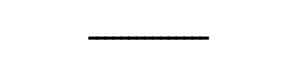 & مطالبة بمده زمنية اضافية \\
\hline 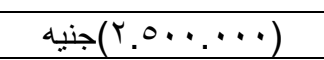 & جنيه & جنيه (Y.YY.rYY) & مطالبة بتكلفة اضافية \\
\hline لم يتم تسويتها & لم يتم تسويتها & لم يتم تسويتها & كيفية التعامل مع المطالبة \\
\hline & & & التعويضات الاضافية فى المدة \\
\hline
\end{tabular}




\begin{tabular}{|l|l|l|c|}
\hline & & & \\
\hline
\end{tabular}

\section{تسوية النزاع (التوفيق / الوسيط ) :}

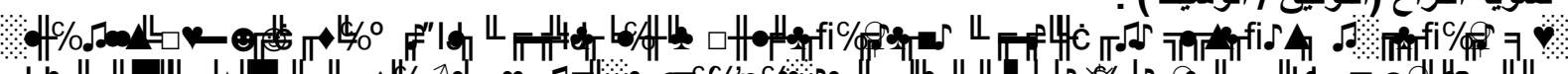
7 政 型

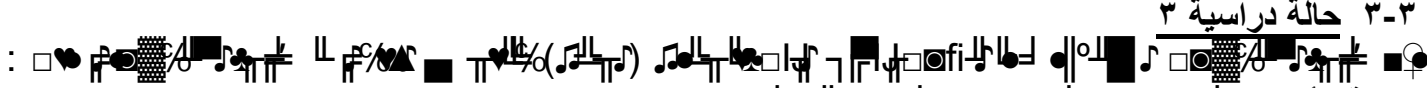

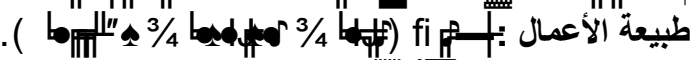

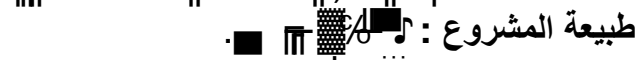
نوع العقد : (lump sum contract )

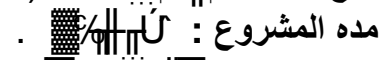

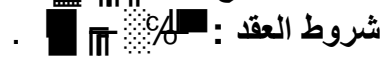
وتتخص المناز عات الخاصة بالمشروع فيما يلى طبقا للجدول رقم (•):

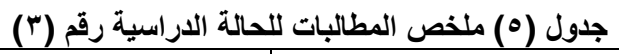

\begin{tabular}{|c|c|c|c|}
\hline المطالبة رقم (ॅ) & المطالبة رقم (ץ) & المطالبة رقم (1) & \\
\hline المالك & المقاول & المقاول & الطرف القائم بالمطالبة \\
\hline 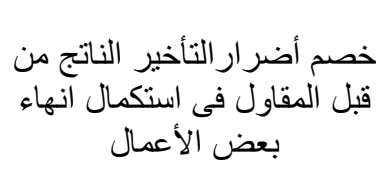 & تغكلفة اضشافيه نتنيجة أو امرة & 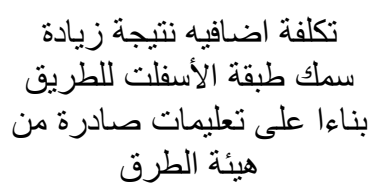 & بيان المطالبة \\
\hline 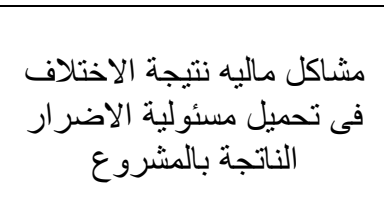 & 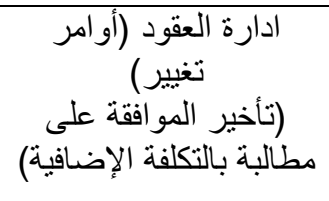 & 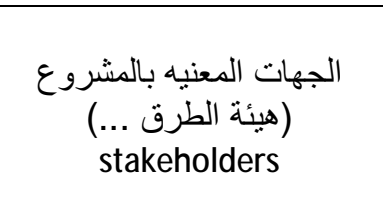 & مصدر المطالبة \\
\hline تكلفة اضافيه & تكلفة اضافية & تكلفة اضافيه & النتائج \\
\hline- & - & 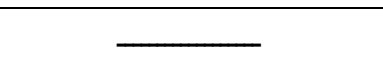 & مطالبة بدده زمنية اضافية \\
\hline (1.1...... (1. جنيه & (YO. r..r) جنيه & (7.7.9.r) & مطالبة بتكلقة اضافية \\
\hline لم ينم تسويتها & لم ينم تسويتها & لم يتم تسويتها & كيفية التعامل مع المطالبة \\
\hline - & - & - & التعويضات الاضافية فى المدة \\
\hline 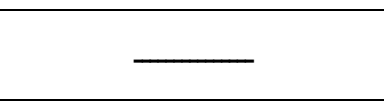 & 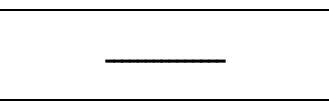 & 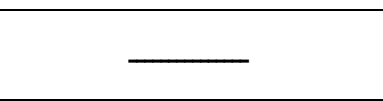 & التعويضات الاضيفةِة فى المدة \\
\hline
\end{tabular}

تسوية النزاع (التحكيم ) :

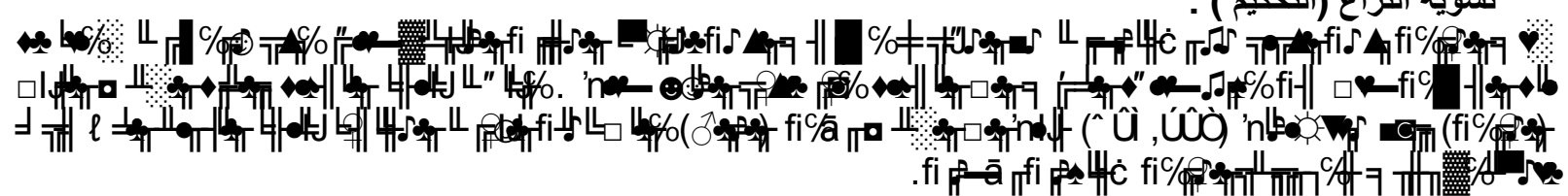

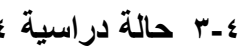

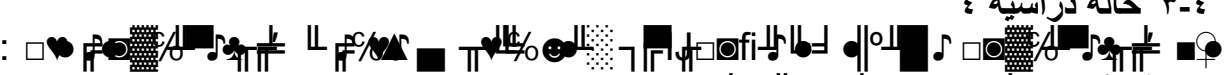
طبيعة الأعمال : fifi

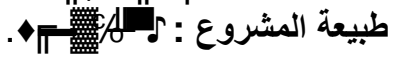


منهجيه لإدارة المطالبات و المناز عات فى مشرو عات التشييد

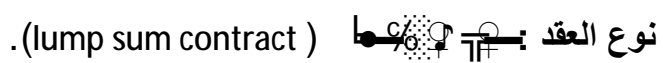

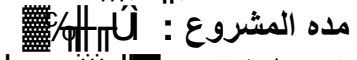

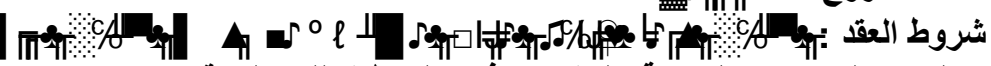
وتتلخص المنازعات الخاصة بالمشروع فيما يلى طبقا للجدول رقم (ه): 


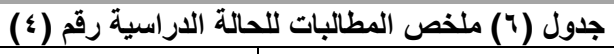

\begin{tabular}{|c|c|c|c|}
\hline المطالبة رقم (r) & المطالبة رقم (Y) & المطالبة رقم (1) & \\
\hline 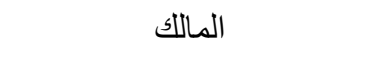 & 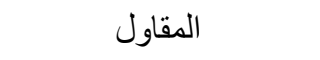 & 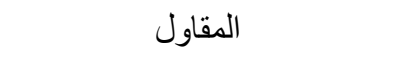 & الطرف القائم بالمطالبة \\
\hline انهاء العقد بسبب اهمال المقاول & تكلفة اضافيه بسبب توقف نتبه & 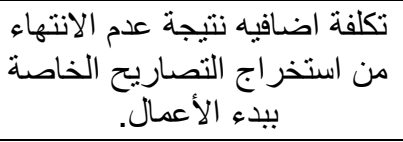 & بيان المطالبة \\
\hline مشاكل ماليه & قهوة قهريه & (معوقات استلام موقع العمل) متعل العل & مصدر المطالبة \\
\hline تكلفة اضافيه & تكلفة ومدة زمنيه اضافية & تكلفة ومدة زمنيه اضافيه & النتائج \\
\hline & 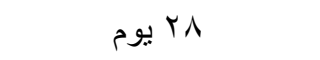 & 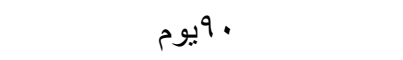 & مطالبة بمده زمنية اضافية \\
\hline جنيه & جنيه (Vo.....) & (I. ( ) جنيه & مطالبة بتكلفة اضافية \\
\hline لم يتم تسويتها & لم يتم تسويتها & لم يتم تسويتها & كيفية التعامل مع المطالبة \\
\hline & & & التعويضات الاضافية فى المدة \\
\hline & & & التعويضات الاضافية فى المدة \\
\hline
\end{tabular}

: تسوية النزاع (التقاضی) :

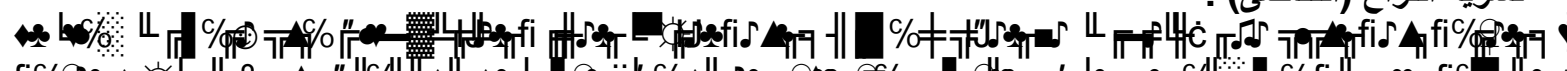

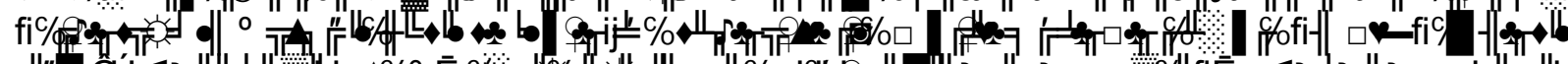

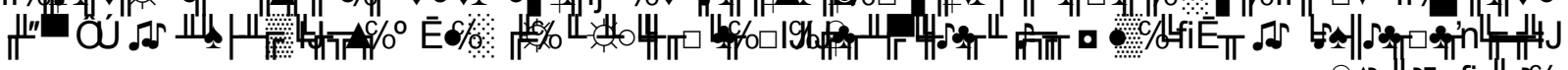

جدول (V) ملخص نتائج النزاعات للحالات الاراسية السابقة

\begin{tabular}{|c|c|c|c|c|}
\hline النتيجة المستفادة & الاجر اعات المتبعة & مصدر المطالبه & نوع العقد & الإراسية \\
\hline أن عمليه التفاوض تعد من أقصر طرق الحس التز اعل & تسويه النزاع) & ادارة العقد- أسباب ماليه - قوهريه & عقد الفيديك (fidic) & (1) \\
\hline 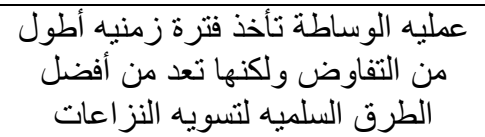 & تسويه النزاعة (الوساطة) & لوائح ونتشريعات العقد - أسباب ماليه- & عقد بتروكيماويات (شروط & $(r)$ \\
\hline 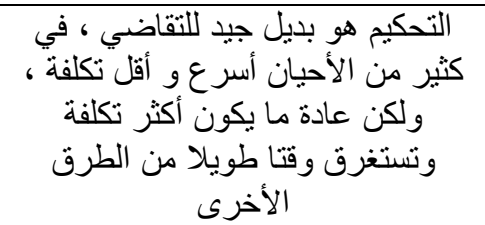 & تسويه النزاع (التحكيم) & الأطر اف العقد - أو امر تغيير بالمشروع & عقد خاص (شروط & $(r)$ \\
\hline 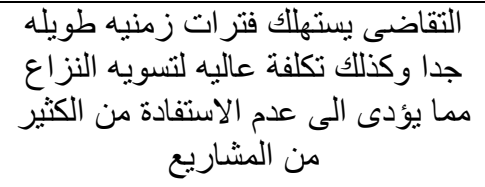 & لم لمتتم تسويته & أسباب ماليه - استام الموقع & 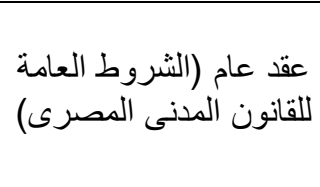 & $(\varepsilon)$ \\
\hline
\end{tabular}

هـ منهجيه الحل لأسباب النزاعات طبقا لمراحل المشروع المختلفة 


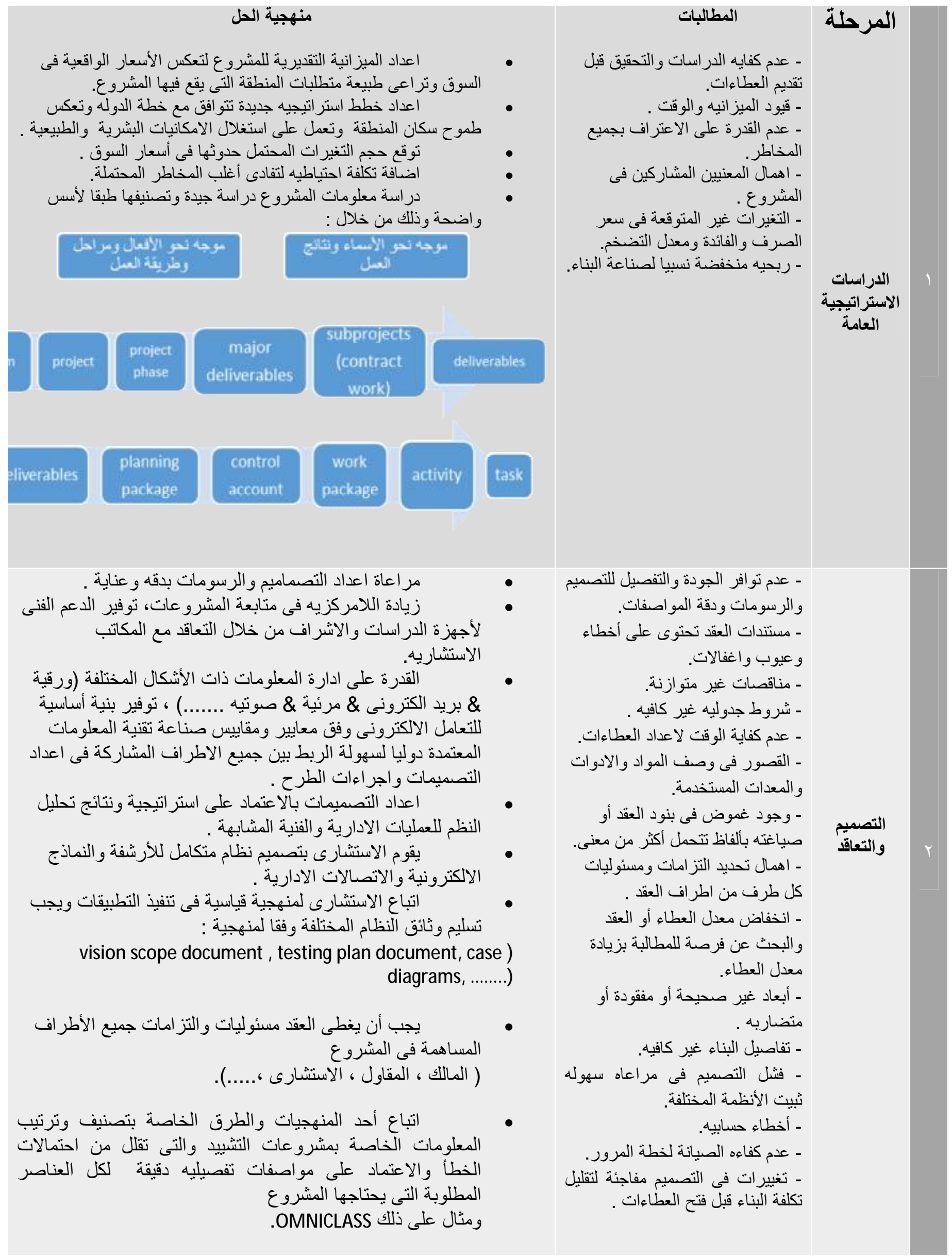


استلام المالك للمشروع بعد التأكد من انتهاء المقاول من جميع الاعمال و الملاحظات طبقا للعقد و الاشتر اطات الفنية الفنية.

سداد جميع مستحقات المقاول بعد الانتهاء من جميع الاعمال الطال

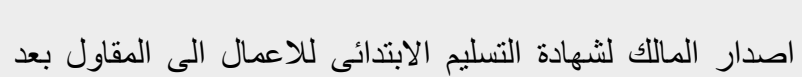

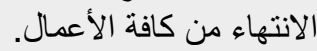
يتم اخلاء الموقع من قبل المقاول وتنظيفة ورد الثئ لأصله قبل

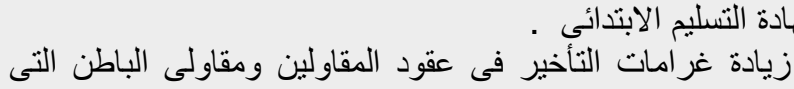

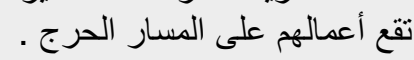

فى حالة وجود أى ملاحظات فى الاعمال يجب اخطار المقاول

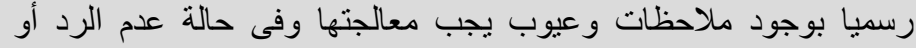

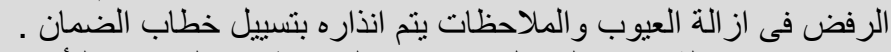

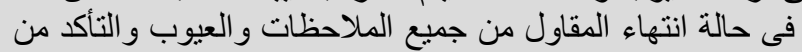

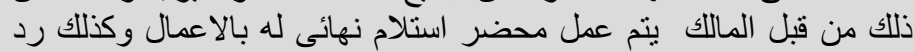

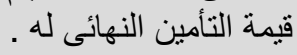

- التأخر أو الامتتاع من استلام المالك الكاول

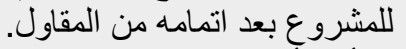
ـ تأخر أو امتتاع المالك من دفع الدفارل الدفعة

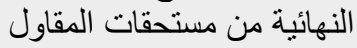

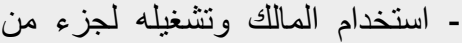
الأعمال قبل انتهاء المقاول من كافة لئة الاعمال - - الاعمال - عدم اخلاء الموقع من قبل المقاول

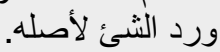

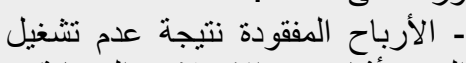
المبنى أثناء فترة الاصنلاح و الصيانة.

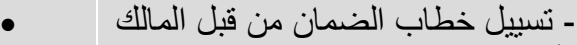
لأى سبب من الاسباب قبل انتهاء مده

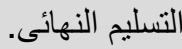

- امتناع المقاول عن تتفيذ أى تعديلات

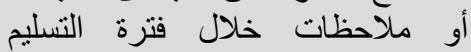

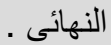
ـ المتتاع المالك عن رد قيمة التأمين

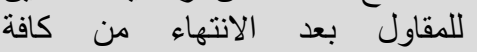

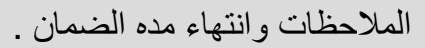

التسليم

النهائى - (الفيم

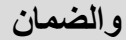

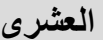




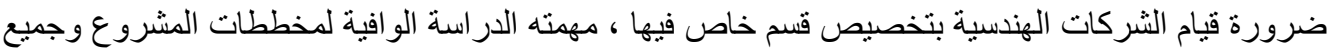
مستنداته من مو اصفات عامة وخاصة وخاتة ودر اسة العقود الهندسية ومتطلباتها حتى تتمكن الثركة من فهم تام

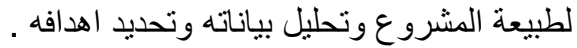

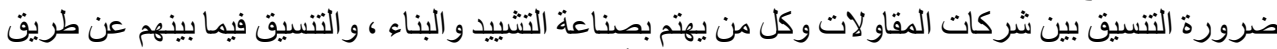

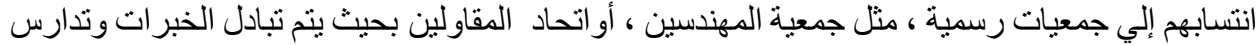
المشاكل التي تو اجهها أثناء التنفيذ.

ضرورة عمل مخططات كافية عن الجودة توضح أهمية تحقيق الجودة لأعمال التشييد و المفاهيم و الأنشطة المتعلقة بذلك.

ضرورة التدقيق في عملية الدر اسة الأولية للمشروع ، من حيث توصيف المشروع بدقة ، وتحديد المتطلبات

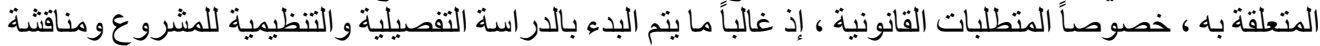

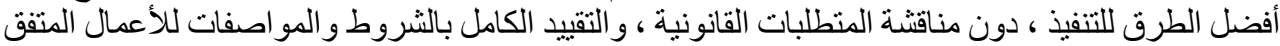
عليها. يجب اختيار المقاولين على أساس العرض الفنى أولا وليس المالى ، و وغالبا هذا مالا يحدث في الواقع ....

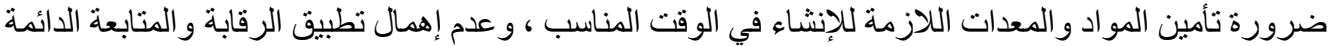

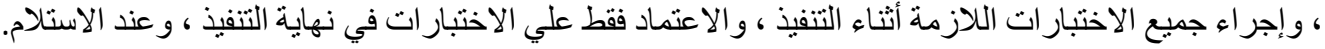

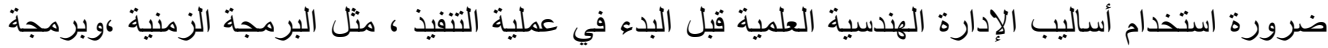

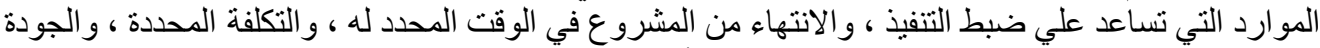

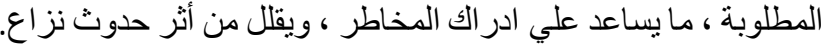

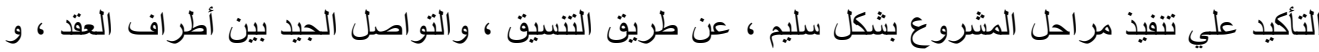

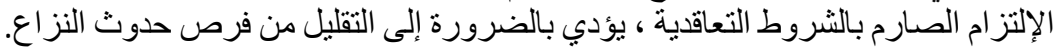

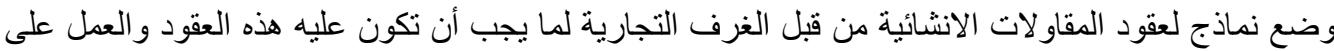

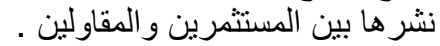

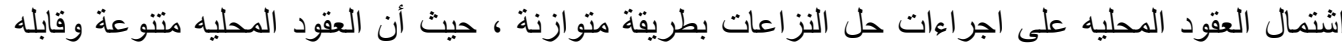

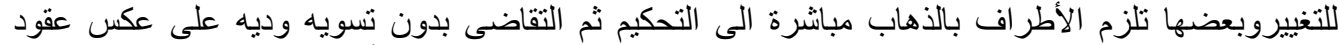
الفيديك التى تناولت تسويه النزاعات بطريقة شامله و التى تعطى الحقوق لجميع الأطر اف الف المتعاقدة.

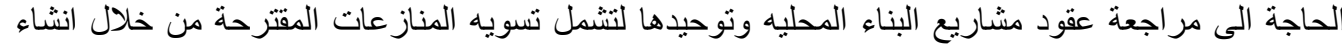

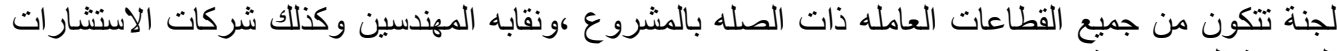
الهندسية المتخصصة. تصنية

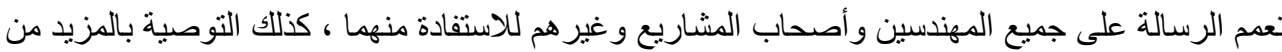
الدر اسه بمثيلاتها من الموضو عات لتغطيه كافة النقاط التى لم يتم استعر اضها لتحقيق لتحفي أقصى استفادة.

وز وزة الاسكان و المر افق و التتمية العمر انية .(9 . . ب). الكود المصرى لادارة مشرو عات التشييد ، القاهرة، الطبعة الأولى .

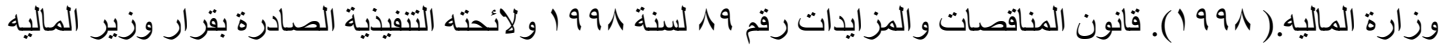

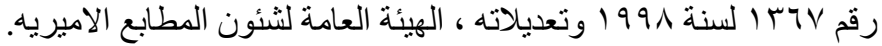

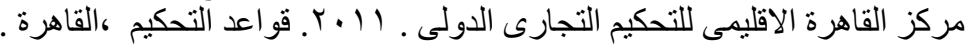

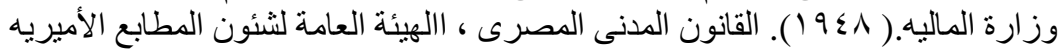

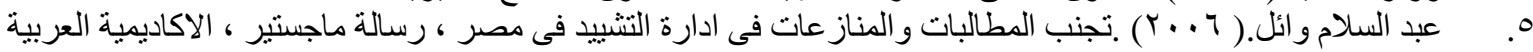

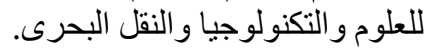

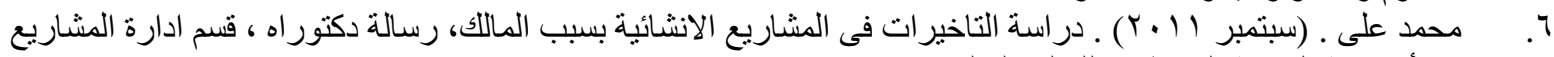
، الأكاديمية العربية البريطانيه للتعليم العالى.

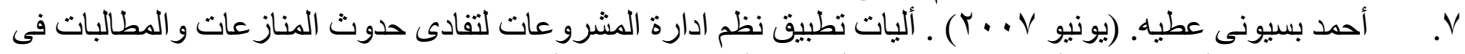

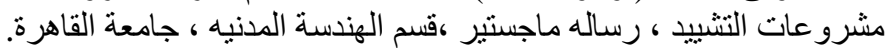

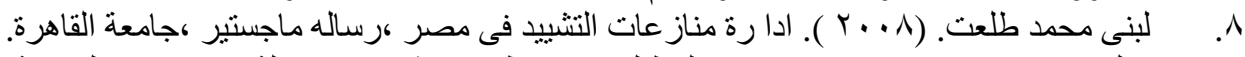

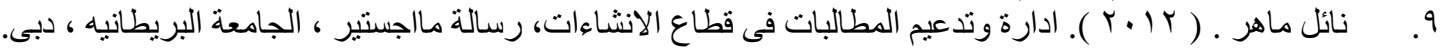

1. Buroog.Basheer. 2012. master degree, faculty of engineering,structural department, cairo university, Egypt.

2. N.Hamzah,M.Akhoiry,N.MTawil. Cause of construction delay. The 2nd Ineternational building control conference 2011 ,procedia engineering 20(2011) 490-495 .

3. Chaitanya.Nityanand.May2015. Management of claims and Disputes in construction. International Journal of Mechanical and civil engineering, (Iosr-Jmce).

4. Manvendra Sinha, Dr. A. S. Wayal. Dispute Causation in Construction Projects. IOSR Journal of Mechanical and Civil Engineering, (IOSR-JMCE) ISSN: 2278-1684, pp. 54-58. 
5. M. Osama Jannadia, Sadi Assaf, A. A. Bubshait, Allam Naji. Contractual Methods for Dispute Avoidance and Resolution (DAR). International Journal of Project Management, 18 (2000), pp. 41-49 .

6. M. M. Kumaraswamy. Conflicts,Claims and Disputes in Construction.Engineering, Construction and Architectural Management, Vol.4, No.2, 1997, pp. 95-111.

7. P. Levin. Construction Contract Claims, Changes \& Dispute Resolution. Second Edition, ASCE Press, Boston, 1998.

8. Ping Ho, S. and Liang Y. (2004). Analytical model for analyzing construction claims and opportunistic bidding. Journal of Construction Engineering and Management

9. , ASCE, Vol. 130, No. 1, pp. 94-104.

10. Fidic.Supplement to First Edition.1999.of conditions of contractfor Building and Engineering works, Designed by the Employer, Geneva,1999.

11. Kartam. S. (1999). Generic methodology for analyzing delay claims. Journal of Construction Engineering and Management, ASCE, Vol. 125, No. 6, pp. 409-419. 